\title{
Erratum to: Atorvastatin Modulates Regulatory T Cells and Attenuates Cerebral Damage in a Model of Transient Middle Cerebral Artery Occlusion in Rats
}

\author{
Ana Lucía Rodríguez-Perea ${ }^{1}$. Johanna Gutierrez-Vargas ${ }^{2}$. \\ Gloria Patricia Cardona-Gómez ${ }^{2}$ C Carlos Julio Montoya Guarin ${ }^{1}$. \\ Mauricio Rojas $^{3,4}$ • Paula Andrea Velilla Hernández ${ }^{1}$
}

Published online: 14 October 2016

(C) Springer Science+Business Media New York 2016

Erratum to: J Neuroimmune Pharmacol (2016) DOI 10.1007/s11481-016-9706-5

The original version of this article unfortunately contained a mistake. The below funding information was missing in Acknowledgments section.

The authors thank the Departamento Administrativo de Ciencia, Tecnología e Innovación (COLCIENCIAS) (Grant 111551928730 207-2010) and the Universidad de Antioquia UdeA (Sostenibilidad and CODI acta 624 de 2012) for its financial support.

The online version of the original article can be found at http://dx.doi. org/10.1007/s11481-016-9706-5

Ana Lucía Rodríguez-Perea

anarope1@gmail.com

1 Grupo Inmunovirología, Facultad de Medicina, Universidad de Antioquia UdeA, Calle 70 No, 52-21 Medellín, Colombia

2 Area de Neurobiología Celular y Molecualr, Grupo de Neurociencias de Antioquia, Facultad de Medicina, Universidad de Antioquia UdeA, Calle 70 No, 52-21 Medellín, Colombia

3 Grupo de Inmunología Celular e Inmunogenética (GICIG), Instituto de Investigaciones Médicas, Facultad de Medicina, Universidad de Antioquia UdeA, Calle 70 No, 52-21 Medellín, Colombia

4 Unidad de Citometría, Facultad de Medicina, Sede de Investigación Universitaria, Universidad de Antioquia UdeA, Calle,

70-52-21 Medellín, Colombia 